\title{
Hollow core waveguide as mid-infrared laser modal beam filter
}

\author{
P. Patimisco, ${ }^{1}$ A. Sampaolo, ${ }^{1,2}$ M. Giglio, ${ }^{1}$ J. M. Kriesel, ${ }^{3}$ F. K. Tittel, ${ }^{2}$ and V. Spagnolo ${ }^{1}$ \\ ${ }^{1}$ Dipartimento Interateneo di Fisica, Università e Politecnico di Bari, CNR-IFN UOS BARI, \\ Via Amendola 173, 70126 Bari, Italy \\ ${ }^{2}$ Department of Electrical and Computer Engineering, Rice University, 6100 Main Street, Houston, \\ Texas 77005, USA \\ ${ }^{3}$ Opto-Knowledge Systems, Inc. (OKSI), 19805 Hamilton Ave., Torrance, California 90502-1341, USA
}

(Received 1 July 2015; accepted 1 September 2015; published online 16 September 2015)

\begin{abstract}
A novel method for mid-IR laser beam mode cleaning employing hollow core waveguide as a modal filter element is reported. The influence of the input laser beam quality on fiber optical losses and output beam profile using a hollow core waveguide with $200 \mu \mathrm{m}$-bore size was investigated. Our results demonstrate that even when using a laser with a poor spatial profile, there will exist a minimum fiber length that allows transmission of only the Gaussian-like fundamental waveguide mode from the fiber, filtering out all the higher order modes. This essentially single mode output is preserved also when the waveguide is bent to a radius of curvature of $7.5 \mathrm{~cm}$, which demonstrates that laser mode filtering can be realized even if a curved light path is required. (C) 2015 AIP Publishing LLC.
\end{abstract}

[http://dx.doi.org/10.1063/1.4930893]

\section{INTRODUCTION}

There are many applications, such as laser-based welding and machining, ${ }^{1,2}$ bio-photonics, ${ }^{3}$ holography, ${ }^{4}$ and spectroscopy $^{5}$ in which mid-IR laser beams with a clean Gaussian spatial mode are highly desirable. In any laser beam, several of the higher order modes can appear to some degree, leading to a beam profile, which does not have perfect spatial quality. The most common method for cleaning up mid-IR beams with poor optical quality is to employ aspheric lens focusing of the laser beam through a pinhole, followed by collimating optics, which collect the light passing through the pinhole. ${ }^{6,7}$ This spatial filtering system may eliminate high-spatial defects of a focused wave, but has the drawback of being highly chromatic. Furthermore, a pinhole is susceptible to misalignment with respect to the laser source, leading to aberrations. In addition, if the pinhole is partially obstructed by dust and contaminations, the optical power is reduced and aberrations are introduced. Pinhole cleaning requires its removal from the optical system and careful realignment when it is replaced. Spatial filtering of light beams has been obtained by using low-refraction-index contrast $3 \mathrm{D}$ photonic crystals. ${ }^{8}$

In this manuscript, we propose a new method for spatial laser mid-IR beam cleaning, in which pinholes are replaced by hollow-core waveguides (HCWs), which act as modal filters. ${ }^{9}$ The optical mode of the fiber with the lowest losses is a hybrid mode characterized by an optical power distribution that follows the fundamental Gaussian mode. Higher order modes have larger losses than the fundamental one when propagating through the fiber. A minimum fiber length will exist that allows only the fundamental mode transmission through the fiber. In this manner, the output end of the fiber appears as a pinhole source of coherent light with an approximate Gaussian intensity distribution. To investigate the influence of the input laser beam quality on fiber losses and output beam profile, we analyzed results obtained by coupling two different lasers emitting at nearly the same wavelength into a $200 \mu \mathrm{m}$-core size HCW. We employed an external cavity quantum cascade lasers (EC-QCL) with a nearly Gaussian beam profile and a distributed-feedback (DFB) QCL source with a low quality beam profile. We demonstrated that the HCW acts as an efficient, mid-IR spatial modal beam filter and an essentially single-mode output beam is obtained in both cases, although, as expected, much higher losses were measured for the DFB QCL source.

\section{WAVEGUIDE COUPLING WITH A GAUSSIAN-LIKE LASER BEAM}

HCWs consist of hollow glass capillary tubes with a metallic/dielectric internal reflecting layer. ${ }^{10}$ The bore size and launching conditions determine the overall optical losses and output beam mode quality. HCWs have extremely high coupling efficiency, no back-reflection, and high power handling capabilities. ${ }^{11-14}$ The HCWs fabrication process is described in detail in Ref. 11. In a first step, we investigated the influence of coupling conditions on the output beam losses and spatial quality when a Gaussian-like laser beam is coupled into a HCW. A schematic description of the HCW-based spatial filtering setup is shown in Fig. 1.

The laser source used was a commercial tunable external cavity EC-QCL system (Daylight Solutions (DLS)), operating at $\lambda=6.24 \mu \mathrm{m}$, with an output power of $80 \mathrm{~mW}$. The beam exiting from the laser is focused on to the waveguide entrance by means of a coupling lens $(1 / 2$ " diameter, focal length $f=50 \mathrm{~mm}$, with an anti-reflection coating centered at $6 \mu \mathrm{m})$. We employed four different HCWs, having the same metallic (Ag)/dielectric (AgI) circular cross-section, internal coatings, and a bore size of $d=200 \mu \mathrm{m}$, but with four different lengths of $12 \mathrm{~cm}, 15 \mathrm{~cm}, 50 \mathrm{~cm}$, and $100 \mathrm{~cm}$. The optical power at the waveguide entrance is measured by means of infrared pyroelectric detector and the output mode profile in the far field condition is recorded by using a pyrocamera 


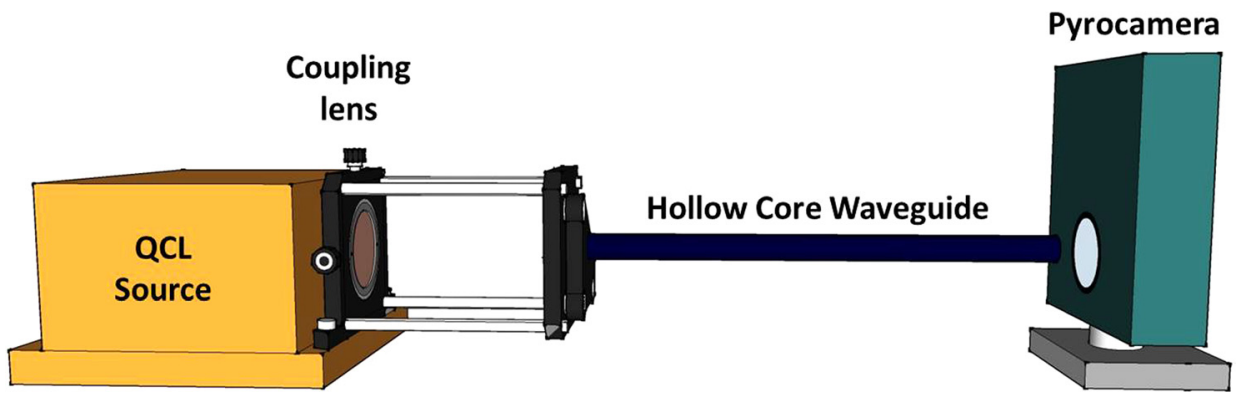

FIG. 1. Schematic of the experimental setup. The laser beam exiting from the EC-QCL source is focused at the waveguide entrance by using a coupling lens. The beam profile at the waveguide exit is acquired by means of an infrared pyrocamera.

(Pyrocam III, Ophir Spiricon) with pixel sizes of $0.085 \times 0.085 \mathrm{~mm}$, mounted at distances $\geq 2.0 \mathrm{~cm}$ from the waveguide output. The coupling lens is fixed to an XYZ translation mount, while the fiber is installed in a mount with 2-angular degrees of freedom, which allows fine tilt adjustments of the HCW entrance with respect to the focused laser beam. The optimal launching condition was found by maximizing the output at the fiber exit. The quality of a laser beam can be expressed in term of its $\mathrm{M}$-squared $\left(\mathrm{M}^{2}\right)$ factor, defined as the ratio between the half-angle beam divergence of the actual laser beam and that of a diffraction-limited Gaussian beam. Thus, a perfect Gaussian beam has an $\mathbf{M}^{2}=1$ and smaller values of $\mathbf{M}^{2}$ are physically not feasible. For non-circularly symmetric beams, the $\mathrm{M}^{2}$ factor can be defined with respect to two directions orthogonal to each other and to the beam axis $\left(\mathrm{M}_{\mathrm{x}}^{2}\right.$ and $\left.\mathrm{M}_{\mathrm{y}}^{2}\right)$, by measuring the half-angle beam divergence of the laser beam in the $x\left(\theta_{x}\right)$ and $y\left(\theta_{y}\right)$ directions. Thus, we have

$$
\begin{aligned}
& M_{x}^{2}=\theta_{x} \cdot \frac{\pi w_{0, x}}{\lambda}, \\
& M_{y}^{2}=\theta_{y} \cdot \frac{\pi w_{0, y}}{\lambda},
\end{aligned}
$$

where $\mathrm{w}_{0, \mathrm{x}}, \mathrm{w}_{0, \mathrm{y}}$ are the beam widths at narrowest point of the collimated laser beam along $\mathrm{x}$ and $\mathrm{y}$ directions, respectively. To measure the beam width, we employed the second-order moment of the beam intensity distribution $I(x, y)$ defined, for both directions $x$ and $y$, as

$$
\begin{aligned}
& w_{x}=2 \sqrt{\frac{\int_{-\infty}^{+\infty} \int_{-\infty}^{+\infty} x^{2} I(x, y) d x d y}{\int_{-\infty}^{+\infty} \int_{-\infty}^{+\infty} I(x, y) d x d y}}, \\
& w_{y}=2 \sqrt{\frac{\int_{-\infty}^{+\infty} \int_{-\infty}^{+\infty} y^{2} I(x, y) d x d y}{\int_{-\infty}^{+\infty} \int_{-\infty}^{+\infty} I(x, y) d x d y}} .
\end{aligned}
$$

The second-order moments are a measure of the variance of the image intensity distribution around the origin. For the evaluation of the moments, we used LabView-based software with the origin of the reference system positioned on the peak intensity of the spatial beam profile. This transformation makes the moment computation independent of the position of the beam reference system. By using the pyrocamera, we collected the laser output-beam profile at different distances from the output window and estimated the beam divergence $\theta_{\mathrm{x}}$ and $\theta_{\mathrm{y}}$. We calculated the second moment width of the beam at different positions of the pyro-camera starting from $4 \mathrm{~cm}$ away from the laser source. The spatial profile of the DLS QCL beam taken $4 \mathrm{~cm}$ away from the laser source is shown in Fig. 2.

The half-angle beam divergences were extracted from the slope of the linear fit to the moments versus distance, and $\mathrm{w}_{0, \mathrm{x}}$ and $\mathrm{w}_{0, \mathrm{y}}$ from their intercept, both for the $\mathrm{x}$ and $\mathrm{y}$ directions. Results are summarized in Table I.

The laser beam exiting from the EC-QCL shows a nearly Gaussian mode profile, as quantified by the calculations of $\mathrm{M}^{2}$ values in both directions. As mentioned previously, when coupling a free-space laser beam into a fiber, an important parameter is the coupling efficiency, defined as the fraction of laser power launched into the fiber. The coupling efficiency can be theoretically estimated via calculation of the overlap integral between the free-space optical mode at the fiber entrance and the waveguide modes. ${ }^{16}$ However, theoretical calculations cannot predict how optical misalignments (such as radial misalignment, defocusing and tilt), lens aberrations, light diffraction, and the quality of the internal surface of the fiber influence the final coupling efficiency. Based on the good $\mathrm{M}^{2}$ values for the EC-QCL, we can assume a pure Gaussian distribution for the beam profile at

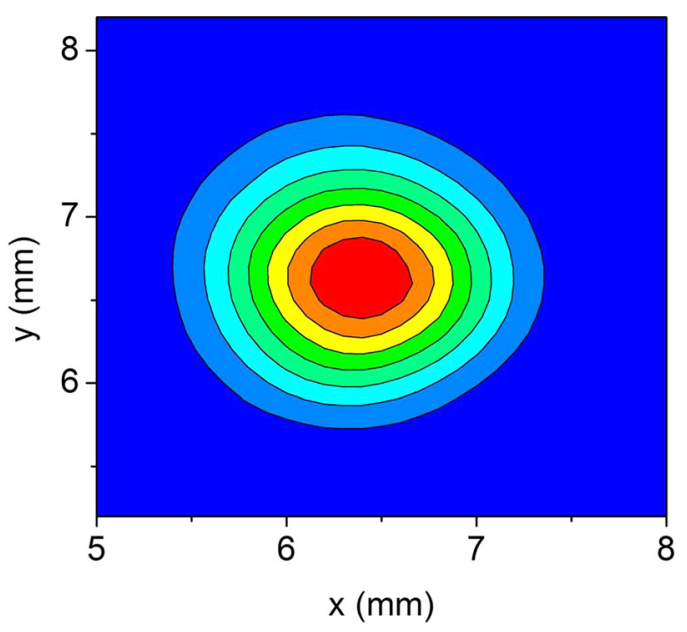

FIG. 2. Two-dimensional far field beam profile of the EC-QCL operating at $\lambda=6.24 \mu \mathrm{m}$, measured at a distance of $4 \mathrm{~cm}$ from the laser source. 
TABLE I. Divergence angles and M-squared values for the DLS QCL in two orthogonal directions.

\begin{tabular}{lcccc}
\hline \hline & $\theta(\mathrm{mrad})$ & $\mathrm{w}_{0}$ & $\lambda /\left(\pi \mathrm{w}_{0}\right)(\mathrm{mrad})$ & $\mathrm{M}^{2}$ \\
\hline x-direction & 1.98 & 1.22 & 1.63 & 1.21 \\
$\mathrm{y}$-direction & 1.88 & 1.20 & 1.65 & 1.14 \\
\hline \hline
\end{tabular}

the waveguide entrance, in both orthogonal directions, $x$ and y to a first approximation. By solving Maxwell's equations considering a cylindrical geometry for the hollow-core fiber, it is feasible to demonstrate that the output beam consists of hybrid modes $\mathrm{HE}_{1 \mathrm{~m}}$ that can be approximated by zero-order Bessel functions. ${ }^{17}$ The $\mathrm{HE}_{11}$ mode is the lowest order mode having a circularly-symmetric, Gaussian spatial profile. According to these considerations, the coupling efficiencies $\eta_{m}$ can be defined for each hybrid mode and the amount of power collected at the waveguide exit $\mathrm{P}_{\mathrm{m}}$ after the propagation inside the fiber of length $L$ is given by

$$
P_{m}=\eta_{m} e^{-2 \alpha_{m} L},
$$

where $\alpha_{m}$ is the fiber attenuation coefficient, which strongly depends on the laser wavelength and the optical properties of the dielectric and metallic layers deposited inside the HCW. ${ }^{15,18}$ The $\eta_{m}$ coupling coefficient for each hybrid modes is a function of the ratio of the focused beam waist at the fiber entrance $\omega_{0}$ and the bore radius $d / 2$ of the waveguide and minimal coupling loss conditions are achieved when $2 \omega_{0} /$ $d=0.64 .{ }^{16}$ Considering that the experimentally measured EC-QCL beam diameter on the coupling lens is $w=2.66 \mathrm{~mm}$, which corresponds to the focused beam waist related to the $f$-number of the coupling lens, using the expression, $f \#=\pi w /$ $2 \lambda$. We can then estimate the required focal length, which can provide low coupling loss conditions. ${ }^{15}$ The best $f$ value result was $44 \mathrm{~mm}$. Thus, in our experiments we employed an $f=50 \mathrm{~mm}$ coupling optics, which leads to a value of $2 \omega_{0} /$ $d \sim 0.74$, close to the optimal value. For this operating condition, the calculated $\alpha_{m}$ and $\eta_{m}$ coefficients for the first waveguide modes are listed in Table II.

Thus, for the optimum conditions, up to $92 \%$ of the input laser light can be coupled into the waveguide with the first $\mathrm{HE}_{11}$ Gaussian-like hybrid mode. The waveguide losses for the $\mathrm{HE}_{11}$ mode are much lower than those for higher order modes. Higher order modes rapidly vanish during their propagation inside a HCW with an optimum fiber length. In this case, only the low-loss $\mathrm{HE}_{11}$ mode survives, making it possible to realize an essentially single mode output with a Gaussian beam profile. By measuring the input and output

TABLE II. Attenuation and coupling coefficients calculated under our experimental conditions.

\begin{tabular}{lcc}
\hline \hline & $\alpha_{\mathrm{m}}(1 / \mathrm{m})$ & $\eta_{\mathrm{m}}$ \\
\hline $\mathrm{m}=1$ & 0.195 & 0.92 \\
$\mathrm{~m}=2$ & 1.03 & $2.40 \times 10^{-4}$ \\
$\mathrm{~m}=3$ & 2.53 & $1.29 \times 10^{-3}$ \\
$\mathrm{~m}=4$ & 4.70 & $4.58 \times 10^{-4}$ \\
$\mathrm{~m}=5$ & 7.54 & $2.12 \times 10^{-4}$ \\
\hline \hline
\end{tabular}

power values at the waveguide entrance and exit, respectively, we calculated the total losses for the HCWs for different fiber lengths. The values obtained, together with the calculated theoretical losses (estimated by using Eq. (3)), are plotted in Fig. 3.

From the slope of the linear fit, we estimated propagation losses of $2.11 \mathrm{~dB} / \mathrm{m}, 20 \%$ higher than the expected value $1.69 \mathrm{~dB} / \mathrm{m}$. The intercept of the linear fit of the experimental data yields the $\eta_{1}$ coupling efficiency if one neglects the small contribution to the coupling losses due to higher order modes. We found that $\eta_{1}=0.78$, which is slightly less than the theoretical value. Such discrepancies can be attributed to fiber imperfections, fabrication, slight optical misalignments, and the presence of scattering losses due to the roughness of the internal fiber reflective coating. ${ }^{15}$ The corresponding far field spatial intensity distribution at the waveguide exit is shown in Fig. 4.

The measured profiles at $\lambda=6.24 \mu \mathrm{m}$ demonstrate that for a Gaussian-like beam and optimal coupling conditions, HCWs with bore sizes of $200 \mu \mathrm{m}$ and fiber lengths of less than $12 \mathrm{~cm}$ allow only the lowest order mode to overcome the losses through the fiber, resulting in a single-mode output beam at the exit. The output with best beam quality was obtained with a fiber length $L=100 \mathrm{~cm}$. It was possible to obtain identical half-angle beam divergences in the $x$ and $y$ directions $\theta_{x}=\theta_{y}=25.1 \mathrm{mrad}$, such that the input beam spatial asymmetry is no longer visible. This value is in excellent agreement with the theoretical half angle beam divergence $\theta_{\text {Theo }}=2.405 \lambda /(\pi d)=23.9 \mathrm{mrad}$, calculated by assuming that only the lowest-order mode propagates. Furthermore, we calculated a $\mathrm{M}^{2}=\theta_{x} / \theta_{\text {Theo }}=1.05$, close to a pure Gaussian beam.

\section{WAVEGUIDE COUPLING WITH A LOW QUALITY LASER BEAM}

The modal properties of the hollow fibers allow their use in filtering out higher-order modes, which have higher losses. Thus, if a poor quality laser beam is launched into a $\mathrm{HCW}$, it can be expected that the guide will filter or strongly

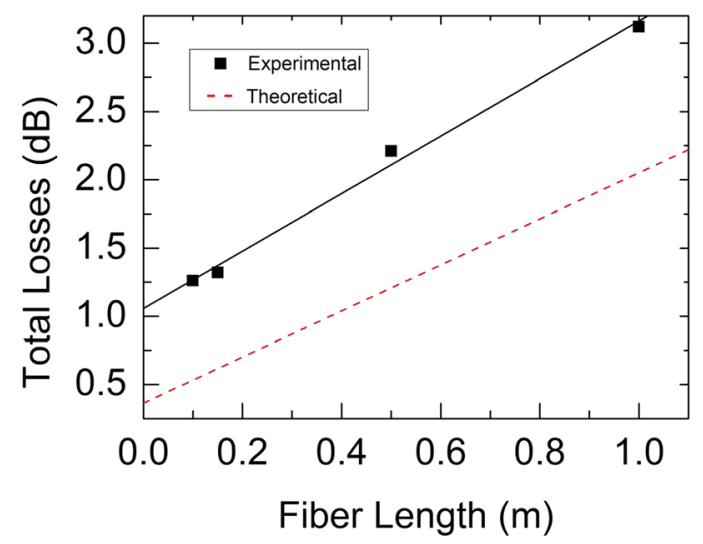

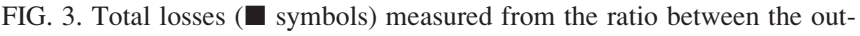
put and input powers while coupling the EC-QCL source in a hollow core waveguide with different lengths. The solid line is the linear fit to the data. The dashed line is the theoretical losses as a function of the fiber length calculated using Eq. (3). 

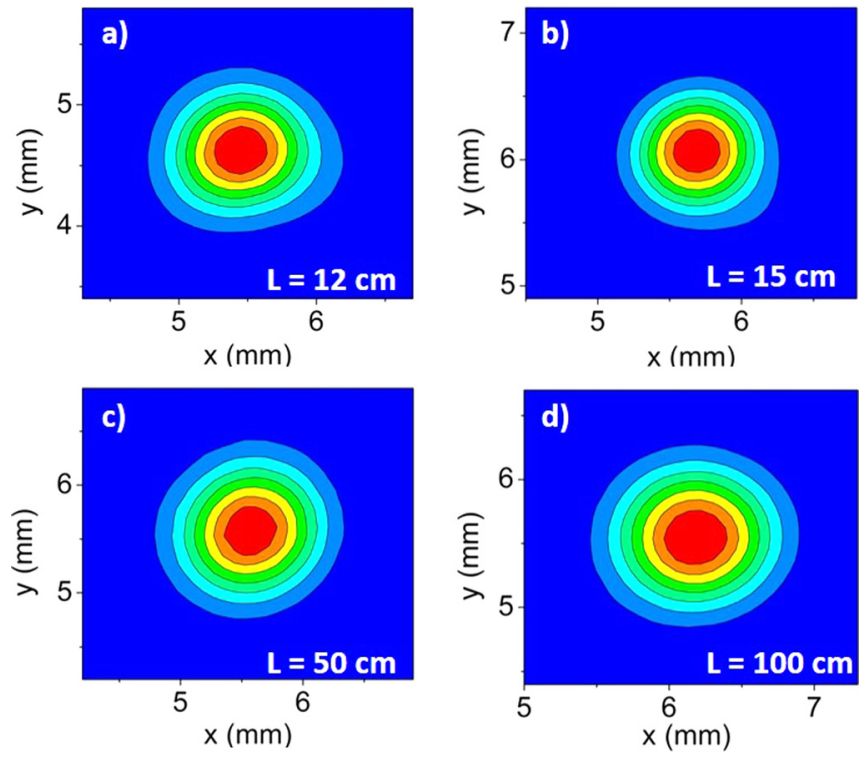

FIG. 4. 2D far field spatial intensity distribution measured upon exiting a $12 \mathrm{~cm} \mathrm{(a),} 15 \mathrm{~cm} \mathrm{(b),} 50 \mathrm{~cm}$ (c), $100 \mathrm{~cm}$ (d) long hollow core waveguides with bore size of $200 \mu \mathrm{m}$, coupled to a EC-QCL source. The beam profiles were acquired by positioning the pyrocamera $2 \mathrm{~cm}$ away from the $\mathrm{HCW}$ exit.

attenuate the higher-order modes as the laser light propagates through the fiber. For this case, the influence of the input beam quality on the propagation losses and on the spatial quality of the fiber output profile is difficult to model. Higher optical losses will occur since the power coupled with higher-order hybrid modes will be absorbed by the fiber. For some applications, such as imaging, ${ }^{4}$ quartz-enhanced photoacoustic sensing, ${ }^{19-21}$ and cavity enhanced spectroscopy, ${ }^{22,23}$ it may be important to improve the beam quality to a Gaussian output mode, even if the optical power is reduced. Based on these considerations, we experimentally investigated the waveguide output mode profile and corresponding optical losses, when an input beam with poor spatial quality is launched into the HCW. The experimental setup employed for this analysis is similar to that shown in Fig. 1, except for the laser source. A continuous-wave (CW), DFB quantumcascade lasers (Alpes Lasers) was used in an ILX mounting (model LDM-4872) equipped with a water cooling system in combination with a short focal lens for laser beam collimation. The laser emission wavelength occurred at $6.24 \mu \mathrm{m}$, identical to that of the EC-QCL, with a maximum power emission of $13 \mathrm{~mW}$ at $-7^{\circ} \mathrm{C}$. To perform a comparison with the results reported above, it is important to employ beams with the same emission wavelength, since the $\alpha_{m}$ attenuation coefficients, and hence the propagation losses, are dependent on the wavelength of the transmitted light. ${ }^{16,18}$ The beam profile acquired at $\sim 2 \mathrm{~cm}$ from the QCL output facet is shown in Fig. 5.

The QCL beam exhibits a poor spatial beam quality along both axes. The far field optical power distribution is characterized by two asymmetric lobes spaced by a node: most of the available optical power $(<95 \%)$ is confined in one lobe. The $\mathrm{M}_{\mathrm{x}}{ }^{2}=3.54$ and $\mathrm{M}_{\mathrm{y}}{ }^{2}=2.74$ values were determined using the LabView code. For comparison with the

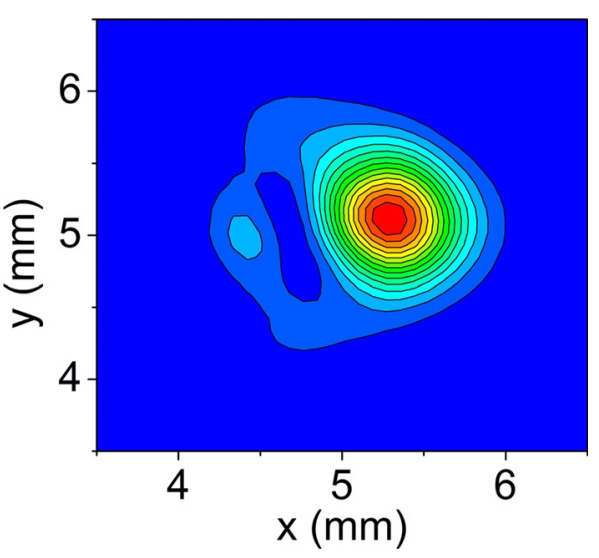

FIG. 5. Measured beam profile of the Alpes Lasers QCL by fixing the pyrocamera $2 \mathrm{~cm}$ far from the QCL.

results obtained for the EC-QCL, we used the same coupling conditions by employing the same optics $(f=50 \mathrm{~mm})$ and fine-tuning the position of the fiber coupling lens in order to obtain an identical beam size $(\sim 2.66 \mathrm{~mm})$. The far field spatial distributions of the fiber output beam, obtained at $2.0 \mathrm{~cm}$ from the fiber end, for the $12 \mathrm{~cm}, 15 \mathrm{~cm}$, and $50 \mathrm{~cm}-10 n g$ fibers are shown in Fig. 6.

An output beam with an excellent symmetric profile was achieved when working with a fiber length of $50 \mathrm{~cm}$. We also verified that when the distance $d$ between the waveguide entrance and the coupling lens differs from the focal length within the Rayleigh range $z_{R}=\pi \omega_{0}{ }^{2} / \lambda \sim 2.8 \mathrm{~mm}$, i.e., $f-z_{R}<d<f+z_{R}$, the output beam quality remained almost unchanged. However, when $d>f+z_{R}$ or $d<f-z_{R}$ (i.e., if the beam is not properly focused into the fiber), the output beam quality decreases, showing spatial distortions of the output beam and the M-squared values increase up to $20 \%$ of the best value (i.e., when $d=f$ ). When $d \gg f+z_{R}$ or $d \ll f-z_{R}$, multimode propagation was observed with related M-squared values of up to 3. By measuring the input and output power values at the waveguide entrance and exit, we calculated total losses of $1.89 \mathrm{~dB}$ for the HCW with fiber length $L=12 \mathrm{~cm}, 2.14 \mathrm{~dB}$ for $L=15 \mathrm{~cm}$ and 4.46 for $L=50 \mathrm{~cm}$. With a $100 \mathrm{~cm}$-long fiber, the optical output power at the waveguide exit was too low and the beam profile could not be measured accurately by the pyrocamera. The measured losses significantly deviate from the theoretical ones, predicted by assuming a pure Gaussian beam coupled at the waveguide entrance (see Fig. 3). The best beam profile was obtained using the $50 \mathrm{~cm}$-long fiber. For this case, we estimated output divergence angles $\theta_{x}=33.0$ mrad and $\theta_{y}=28.6 \mathrm{mrad}$, and correspondingly $\mathrm{M}_{\mathrm{x}}{ }^{2}=1.37$ and $\mathrm{M}_{\mathrm{y}}{ }^{2}=1.19$. Thus, our results confirm that the spatial quality of the laser beam can be improved by means of HCW filtering and it is possible to obtain a Gaussian-like spatial beam profile even if the input laser beam quality is poor. The fiber length is a crucial parameter since the fiber must be long enough to effectively absorb higher order modes and hence obtain propagation of essentially the lowest order mode and thus a nearly single mode output beam. This is evident by observing the beam profiles acquired for both the $12 \mathrm{~cm}$ and $15 \mathrm{~cm}$-long fibers. A slight spatial distortion is 

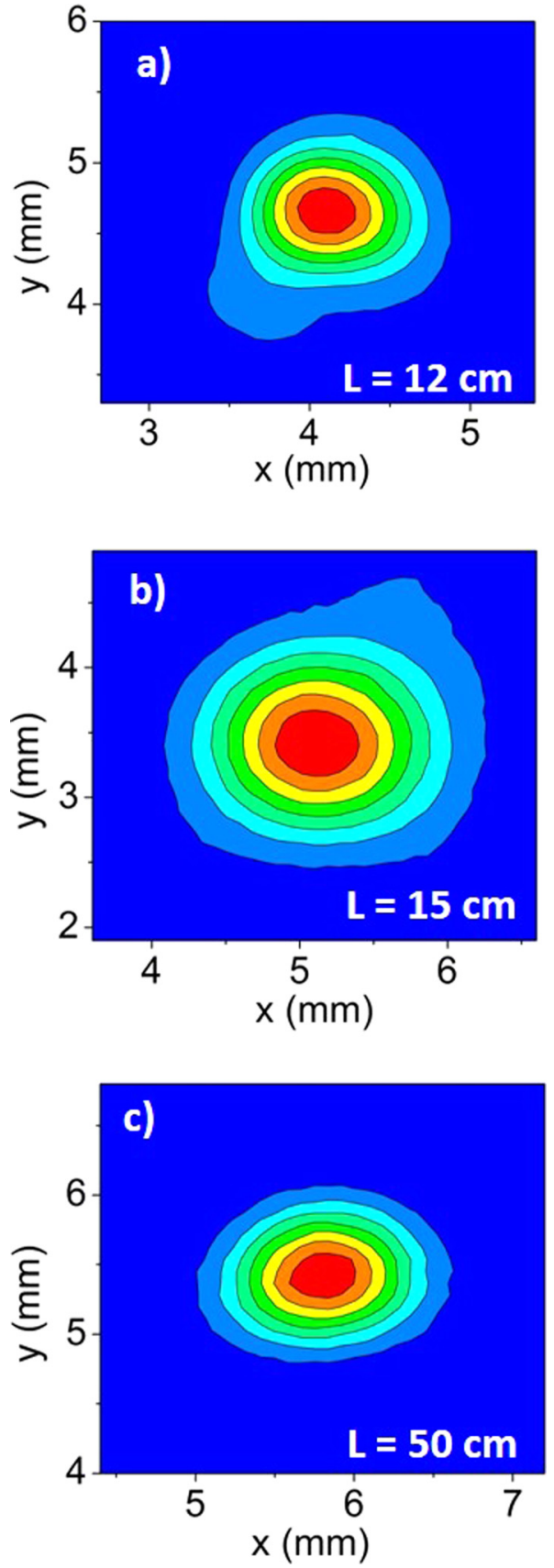

FIG. 6. Measured far field spatial intensity distribution measured upon exit-

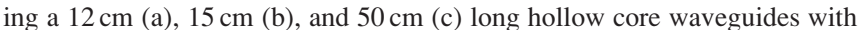
a bore size of $200 \mu \mathrm{m}$, coupled to an Alpes Lasers CW, DFB QCL source. The beam profiles have been acquired by positioning the pyrocamera $2 \mathrm{~cm}$ away from the HCW exit.

visible and is due to the presence of high order modes that are not completely quenched when using HCWs of length $\leq 15 \mathrm{~cm}$. In Table III, we summarize the $\mathrm{M}^{2}$ results measured for both EC-QCL and DFB QCL sources, at the input and output of the HCW.

We also investigated the influence of fiber bending on the waveguide performances, i.e., the beam quality and total losses at different radii of curvature. For this analysis, we selected the fiber having a length of $50 \mathrm{~cm}$. The measurements were made by keeping straight the two ends of the fiber and bending a center part with a fixed length of $25 \mathrm{~cm}$ to a uniform bending radius. ${ }^{15}$ In Fig. 7 , we reported three
TABLE III. M-Squared values measured for EC-QCL and DFB-QCL at the entrance and output of the HCW.

\begin{tabular}{lccccc}
\hline \hline & \multicolumn{2}{c}{ EC-QCL } & & \multicolumn{2}{c}{ DFB-QCL } \\
\cline { 2 - 3 } \cline { 5 - 6 } & Input beam & Output beam nn & & Input beam & Output beam \\
\hline $\mathrm{M}_{\mathrm{x}}{ }^{2}$ & 1.21 & 1.05 & & 3.54 & 1.37 \\
$\mathrm{M}_{\mathrm{y}}{ }^{2}$ & 1.14 & 1.05 & & 2.74 & 1.19 \\
\hline \hline
\end{tabular}
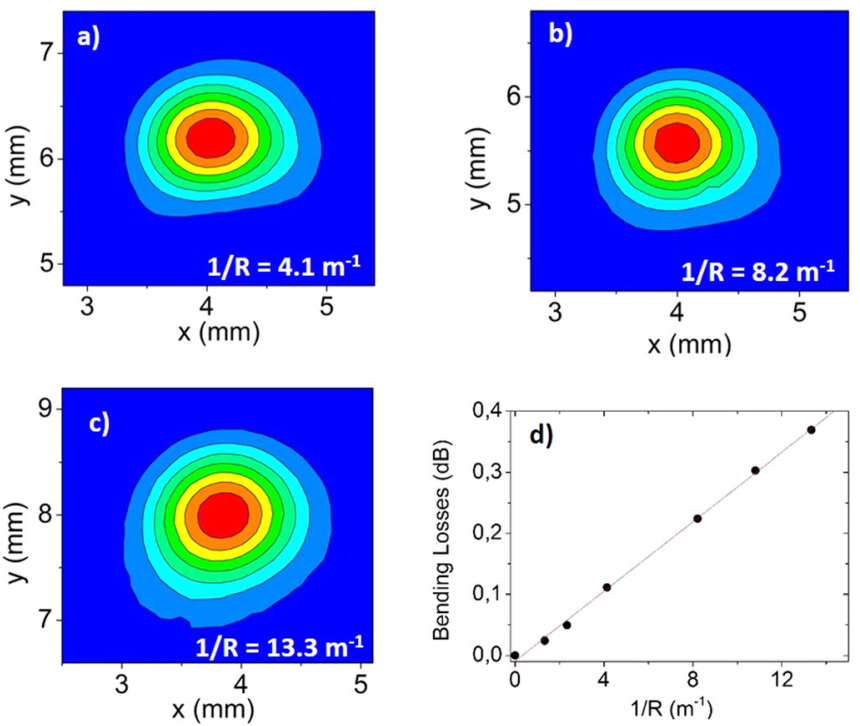

FIG. 7. Output beam profiles acquired at $\sim 2 \mathrm{~cm}$ away for $50 \mathrm{~cm}$-long $\mathrm{HCW}$ coupled with Alpes Lasers CW, DFB, QCL with the coupling lens of $\mathrm{f}=50 \mathrm{~mm}$, when the $\mathrm{HCW}$ is bent to uniform bending radii of $24.4 \mathrm{~cm}$ (a), $12.2 \mathrm{~cm}$ (b), and $7.52 \mathrm{~cm}$ (c). (d) Measured bending losses plotted as a function of the radius of curvature.

representative far field spatial beam profiles acquired at beam radii of $7.52 \mathrm{~cm}, 12.2 \mathrm{~cm}$, and $24.4 \mathrm{~cm}$.

Bending losses, calculated by subtracting the straight losses to the total ones, as a function of the inverse of radius of curvature are also reported in Fig. 7(d) and increase linearly to $1 / R$, as expected. ${ }^{24}$ The essentially single mode behavior is preserved with radii of curvature down to $7.5 \mathrm{~cm}$ and bending losses up to $0.38 \mathrm{~dB}$. This further demonstrates the efficacy of employing $\mathrm{HCW}$ as a laser mode filter, even when bending of the beam is required.

\section{CONCLUSIONS}

This paper reported a novel method for absorbing highorder mid-IR laser modes and provides effectively single mode operation (i.e., the lowest order mode), even if lasers with poor input beam quality are employed. Mode filtering is realized by using HCW with bore size of $200 \mu \mathrm{m}$. The laserwaveguide coupling conditions are crucial in determining the quality of the fiber output beam and the optical losses. In our experiments, the optical alignment was optimized to couple the input beam power into the first Gaussian-like waveguide mode, $\mathrm{HE}_{11}$. Such an approach also reduces the coupling and propagation losses through the fiber. A bending beam loss analysis demonstrates the possibility to perform laser mode filtering even if waveguide curving becomes 
necessary for a specific application. Finally note that using the $200-\mu \mathrm{m}$ core HCW, we recently demonstrate effectively single-mode operation with mid-IR QCLs working in the wavelength range $5.1-10.5 \mu \mathrm{m},{ }^{15}$ thus good mode filtering results can be expected over this entire wavelength range.

\section{ACKNOWLEDGMENTS}

The authors from Dipartimento Interateneo di Fisica di Bari acknowledge financial support from two Italian research Project Nos. PON02 00675 and PON02 00576.

${ }^{1}$ A. Volpe, F. Di Niso, C. Gaudiuso, A. De Rosa, R. M. Vazquez, A. Ancona, P. M. Lugarà, and R. Osellame, Opt. Express 23, 4114 (2015).

${ }^{2}$ A. Ancona, S. Doring, C. Jauregui, F. Roser, J. Limpert, S. Nolte, and A. Tunnermann, Opt. Lett. 34, 3304 (2009).

${ }^{3}$ J. L. West and N. J. Halas, Annu. Rev. Biomed. Eng. 5, 285 (2003).

${ }^{4}$ S. De Nicola, P. Ferraro, S. Grilli, L. Miccio, R. Meucci, P. K. BuahBassuah, and F. Tito Arecchi, Opt. Commun. 281, 1445 (2008).

${ }^{5}$ P. Patimisco, G. Scamarcio, F. K. Tittel, and V. Spagnolo, Sensors 14, 6165 (2014).

${ }^{6} \mathrm{P}$. Hariharan, in Optical Holography, edited by P. L. Knight (Cambridge University Press, Cambridge, UK, 1996).

${ }^{7}$ V. Kermene, A. Saviot, M. Vampouille, B. Colombeau, C. Froehly, and T. Dohnalik, Opt. Lett. 17, 859 (1992).

${ }^{8}$ L. Maigyte, T. Gertus, M. Peckus, J. Trull, C. Cojocaru, V. Sirutkaitis, and K. Staliunas, Phys. Rev. A 82, 043819 (2010).
${ }^{9}$ J. M. Kriesel, G. M. Hagglund, N. Gat, V. Spagnolo, and P. Patimisco, SPIE Proc. 8993, 89930V (2013).

${ }^{10} \mathrm{~J}$. A. Harrington, Fiber Integr. Opt. 19, 211 (2000).

${ }^{11}$ J. A. Harrington, in Infrared Fibers and Their Applications, edited by J. A. Harrington (SPIE, Bellingham, WA, 2004).

${ }^{12}$ C. M. Bledt, J. A. Harrington, and J. M. Kriesel, Appl. Opt. 51, 3114 (2012).

${ }^{13}$ P. Patimisco, V. Spagnolo, M. S. Vitiello, A. Tredicucci, G. Scamarcio, C. M. Bledt, and J. A. Harrington, Appl. Phys. B 108, 255 (2012).

${ }^{14}$ P. Patimisco, V. Spagnolo, M. S. Vitiello, A. Tredicucci, G. Scamarcio, C. M. Bledt, and J. A. Harrington, Sensors 13, 1329 (2013).

${ }^{15}$ A. Sampaolo, P. Patimisco, J. M. Kriesel, F. K. Tittel, G. Scamarcio, and V. Spagnolo, Opt. Express 23, 195 (2015).

${ }^{16}$ R. Nubling and J. A. Harrington, Opt. Eng. 37, 2454 (1998).

${ }^{17}$ E. A. J. Marcantili and R. A. Schmeltzer, Bell Syst. Tech. J. 43, 1783 (1964).

${ }^{18}$ R. George and J. A. Harrington, Appl. Opt. 44, 6449 (2005).

${ }^{19}$ V. Spagnolo, P. Patimisco, S. Borri, G. Scamarcio, B. E. Bernacki, and J. Kriesel, Opt. Lett. 37, 4461 (2012).

${ }^{20}$ V. Spagnolo, P. Patimisco, S. Borri, G. Scamarcio, B. E. Bernacki, and J. Kriesel, Appl. Phys. B 112, 25 (2013).

${ }^{21}$ M. Siciliani de Cumis, S. Viciani, S. Borri, P. Patimisco, A. Sampaolo, G. Scamarcio, P. De Natale, F. D'Amato, and V. Spagnolo, Opt. Express 22, 28222 (2014).

${ }^{22}$ G. Gagliardi and H. P. Loock, in Cavity-Enhanced Spectroscopy and Sensing, edited by G. Gagliardi and H. P. Loock (Springer, London, 2014).

${ }^{23}$ P. Patimisco, S. Borri, A. Sampaolo, H. E. Beere, D. A. Ritchie, M. S. Vitiello, G. Scamarcio, and V. Spagnolo, Analyst, Royal Society of Chemistry 139, 2079 (2014).

${ }^{24}$ M. Miyagi, Appl. Opt. 20, 1221 (1981). 